\title{
Synthesis of Highly Tetrahedral Amorphous Carbon by Mixed-Mode HiPIMS Sputtering
}

\author{
R. Ganesan, ${ }^{1}$ D.G. McCulloch, ${ }^{2}$ N.A. Marks, ${ }^{3}$ M.D. Tucker, ${ }^{4}$ J.G. Partridge, ${ }^{2}$ M.M.M. Bilek, ${ }^{1}$ and D.R. \\ McKenzie ${ }^{1}$ \\ 1) School of Physics, The University of Sydney, Sydney NSW 2006, Australia \\ ${ }^{2)}$ School of Applied Sciences, RMIT University, GPO Box 2476, Melbourne VIC 3001, \\ Australia \\ ${ }^{3)}$ Nanochemistry Research Institute, Curtin Institute for Computation, and Department of Physics and Astronomy, \\ Curtin University, Perth WA 6102, Australia \\ 4) Department of Physics and Astronomy, Curtin University, Perth WA 6102, \\ Australia
}

(Dated: 21 August 2015)

Tetrahedral amorphous carbon films with an $s p^{3}$ content of $80 \%$ have been produced by high power impulse magnetron sputtering (HiPIMS) operating in a mixed sputtering/arc mode. In this mode, short-lived cathode spots form in the magnetic racetrack and produce large numbers of carbon ions. The spots move rapidly, inhibiting the formation of macroparticles. An argon pressure below 2.5 mTorr was critical for obtaining films with high $s p^{3}$ content, high stress, large Tauc gap and symmetrical Raman spectra, and all four quantities were strongly correlated.

Tetrahedral amorphous carbon ( $t a-\mathrm{C})$ has a noncrystalline network structure with a majority of carbon atoms in the $s p^{3}$ hybridisation and a low hydrogen content. One of the hardest known glasses, ta-C is optically transparent, ${ }^{1,2}$ has a low friction coefficient, ${ }^{3}$ a high spin density $^{4}$ and is a semiconductor that can be doped $n$ type. ${ }^{5}$ Current applications of $t a-C$ include corrosion barriers in magnetic hard drives, wear-resistant coatings for cutting tools and scratch-resistant layers on optical components. Potential new electronic uses of ta-C include a non-volatile memory and anti-fuse technology. The key to synthesizing highly tetrahedral amorphous carbon films from $\mathrm{C}$ ion fluxes is the energy distribution of the depositing species which is found to be centred around $100 \mathrm{eV}$ for the highest $s p^{3}$ fractions. ${ }^{2}$ Three methods have been typically used for $t a-\mathrm{C}$ synthesis from carbon ion fluxes: cathodic arc deposition, pulsed laser deposition and mass selected ion beam deposition. Cathodic arc deposition is most commonly used as a coating technology but has the disadvantage that it produces macroparticles formed in the explosive cathode spot plasma which can only be removed with a filter.

Sputtering is widely used as a coating technology but it has not been applied in commercial ta-C deposition as its credentials as a method for forming high $s p^{3}$ carbon films are not firmly established. Although there are reports of ta-C synthesis by sputtering-based methods, ${ }^{6-8}$ the materials characterization evidence is not universally consistent and there are conflicting reports on the optimal conditions for tetrahedral bonding. For the most part, studies using DC or RF sputtering do not find any operating conditions that yield high $s p^{3}$ fractions. ${ }^{9-11}$ This is a consequence of the sputtering process itself, in which the depositing flux mainly comprises low energy carbon neutrals and sputter-gas ions, typically argon. The development of High Power Impulse Magnetron Sputtering (HiPIMS) offers a new direction in which the physics of the deposition process differs greatly to conventional sputtering. With HiPIMS the instantaneous current is several orders of magnitude higher, leading to increased ionization of the sputtered species. There have been multiple attempts ${ }^{11-19}$ to employ HiPIMS to synthesize $t a-\mathrm{C}$, but none have achieved $s p^{3}$ fractions above $45 \%$, probably because HiPIMS ionizes carbon less effectively than it does other elements. One possible exception is a study ${ }^{20}$ employing neon instead of argon as the sputter gas: densities similar to $t a-\mathrm{C}$ were reported, but no other film properties were determined.

In this work we show how a mixed cathodic arc/sputtering mode ${ }^{21}$ can be employed in a HiPIMS system to enable deposition of highly tetrahedral amorphous carbon films. In this mode, short-lived cathodic arcs form on the target, boosting the fraction of ionized carbon. The arcs do not require triggering, instead arising due to the runaway increase in current. Confirmation of the tetrahedral nature of the films is provided by comprehensive characterization of electronic, optical, vibrational and mechanical properties.

We implemented HiPIMS in two different magnetron sputtering systems. The first is an AJA system using a 3 inch AJA A330 magnetron sputtering source and with a base vacuum of $10^{-7}$ Torr. The second is a custom system using a 3 inch Meivac MAK magnetron, with a base vacuum of $<10^{-7}$ Torr. In both cases, RUP7 GBS Elektronik power supplies were used to drive the magnetron with $\sim 640 \mathrm{~V}$ pulses of maximum length $210 \mu$ s at a rate of $100 \mathrm{~Hz}$. These power supplies are capable of delivering peak currents of at least $300 \mathrm{~A}$. The targets used were $6 \mathrm{~mm}$ thick high-density graphite of $99.99 \%$ purity.

Figure 1(a) shows a typical current trace in HiPIMS mixed-mode. At first, the current to the magnetron increases in the usual manner of a HiPIMS discharge. Once the current reaches about $200 \mathrm{~A}$, the slope of the current trace increases abruptly; this transition is the point at 
which an arc ignites on the target surface. Around $10 \mu \mathrm{s}$ afterwards, the pulse is terminated when either the preset current or time limit is reached. The ion current to the negatively biased substrate exhibits a similar trend, with an abrupt increase after the arc ignites, suggesting that the mixed-mode arc spots are a strong source of carbon ion emission. Measurements of 100 pulses showed a time delay of $12.3 \pm 0.1 \mu$ s between the the peak magnetron current and the peak substrate current. Based on the $22 \mathrm{~cm}$ target-substrate distance, this delay converts to an energy of $20 \mathrm{eV}$ if the ions are not scattered by the process gas. As the mean free path of fast $\mathrm{C}$ in $\mathrm{Ar}$ at $2.25 \mathrm{~m}$ Torr is of order $10 \mathrm{~cm}$, some scattering will occur and so this energy should be interpreted as a minimum value for some of the ions emitted. For comparison, the most likely energy of carbon ions emitted by a carbon cathodic arc is $18.7 \mathrm{eV}^{22}$

Figure 1(b) shows time-resolved optical emission spectra collected before and after arc ignition. Light was collected side-on to the discharge using a lens focussed on a region immediately above the target racetrack. This light was analysed using a grating spectrometer fitted with an intensified charge-coupled-device (CCD) camera. Measured relative to $\mathrm{Ar}^{+}$, the intensity from $\mathrm{C}^{+}$emission increases substantially during an arc, while the intensity from neutral Ar decreases. The spectrum after arc ignition was acquired at the end of the pulse with a gate time of $250 \mathrm{~ns}$; the other spectrum was acquired $60 \mu$ s earlier. In both cases, spectra from 50 pulses were averaged. The spectra were normalized to the intensity of the $514.18 \mathrm{~nm}$ Ar II line, as this has an upper energy close to that of the carbon ion lines. The isolated carbon ion (C II) lines at 513.29 and $513.33 \mathrm{~nm}$ provide the clearest evidence of the increase in carbon ionization during the arc. Further evidence is provided by the C II lines at $514.35,514.52$ and $515.11 \mathrm{~nm}$, although they are partially obscured by nearby argon neutral (Ar I) and ion (Ar II) lines.

Figure 1(c) is a composite image showing 100 arc spots on the target surface. The image was assembled from photographs of individual pulses where the shutter was open for the entirety of the pulse. In order to show the arcs, only the red channel of each image was used when the images were combined, while the close-up views in the insets show the full-colour photographs. Bright spots are uniformly distributed over the race track region. The spots do not occur if the discharge is terminated prior to the initiation of an arc, and there is only one spot per pulse. The spots move rapidly and frequently divide, producing fractal-like paths as shown in inset (i). The spots move around the target in a "retrograde" motion well-known in cathodic arcs ${ }^{23}$ whereby the spot motion is counter to $\mathbf{j} \times \mathbf{B}$, where $\mathbf{j}$ is the conventional current density and $\mathbf{B}$ is the magnetic field. Their fractal-like structure is also well-known in cathodic arcs, and is a likely result of spot splitting due to the increase in current during the lifetime of the spot and the known maximum current carried by an individual arc of $\sim 200 \mathrm{~A}$ per spot. ${ }^{24}$ The high velocity and short lifetime of the
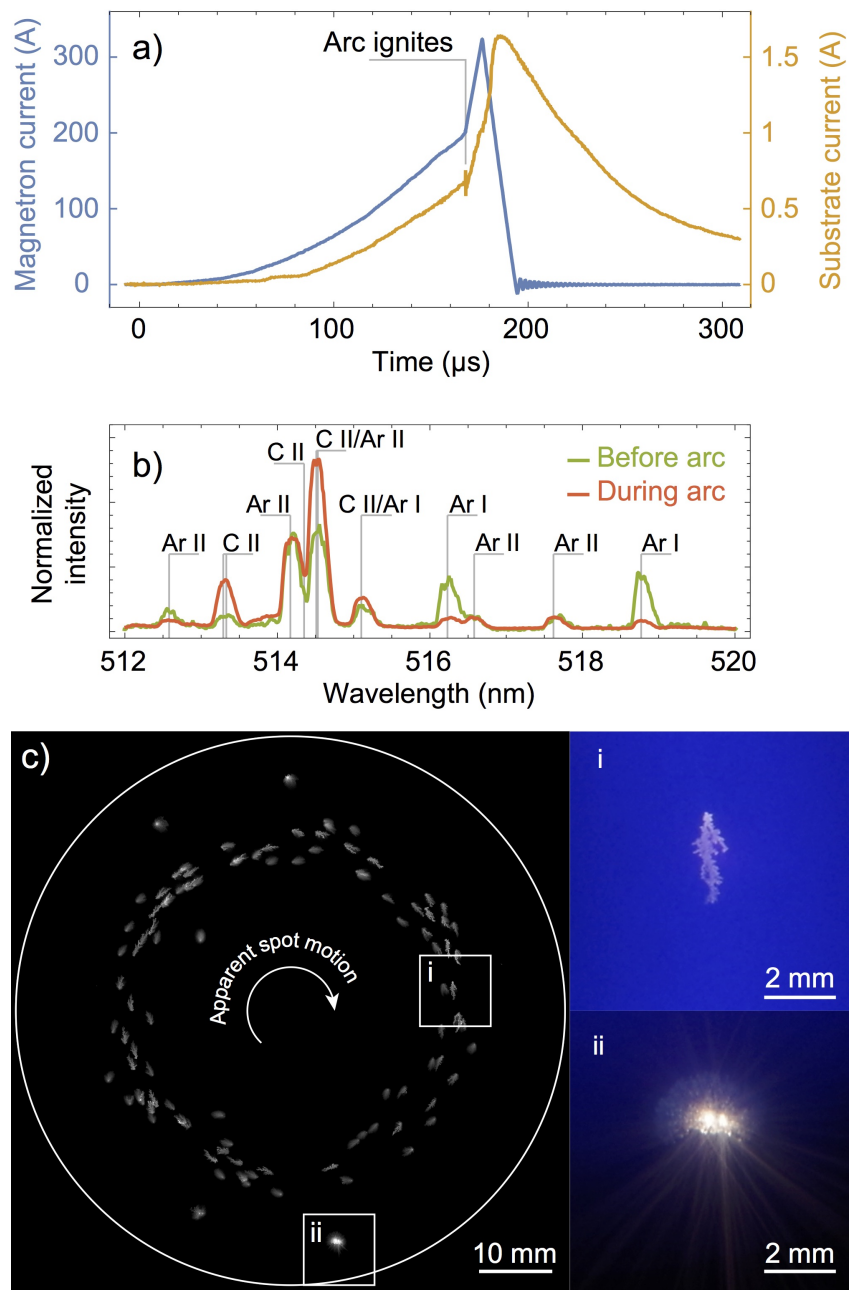

FIG. 1. (a) Typical magnetron current (blue) and substrate current (gold) traces for a mixed-mode HiPIMS pulse. The substrate was biased to $-100 \mathrm{~V}$ dc. (b) Optical emission spectra (each accumulated over 50 pulses) collected before and after the triggering of the arc. (c) Composite image showing photographs of 100 arcs on the surface of the magnetron target. The large circle shows the target edge. Inset (i) is an enlarged view of a typical arc located in the racetrack. Inset (ii) is an enlarged view of a less typical arc that ignited outside the racetrack and ejected incandescent macroparticles. All data shown was collected at an Ar pressure of 2.25 mTorr.

arc spots prevents excessive dissipation of energy at a single point on the target and so largely avoids the explosive process of macroparticle generation observed in cathodic vacuum arc carbon sources. Such macroparticle formation occurs occasionally when the arc ignites away from the racetrack where it is not subject to $\mathbf{j} \times \mathbf{B}$ steering. An example of such an event, showing incandescent macroparticle traces, is shown in inset (ii). Rotating ionization zones, or spokes, are a well-known example ${ }^{25}$ of spatial inhomogeneity in HiPIMS discharges, but the arcs appear to be a separate phenomenon. Compared to the arcs, the ionization zones are substantially larger, move with a much higher velocity, and circulate around 
the magnetron in the opposite direction.

Thin films were deposited onto negatively biased $(-100 \mathrm{~V})$ silicon substrates using mixed-mode HiPIMS for Ar pressures of 1.75-5 mTorr. All films were deposited on the AJA system using a pulse length of $210 \mu \mathrm{s}$. With this pulse length the probability of arc ignition is $100 \%$. A deposition time of 60 minutes resulted in film thicknesses of 70-90 $\mathrm{mm}$ and so the deposition rate was $1.2-1.5 \mathrm{~nm} / \mathrm{min}$. Fourier Transform Infra Red (FTIR) spectra showed no absorption in the region of $3000 \mathrm{~cm}^{-1}$ corresponding to $\mathrm{C}-\mathrm{H}$ group absorption, indicating that the hydrogen concentration is below the sensitivity limit of the measurement (1\%).

Figure 2(a) shows the electron energy loss (EELS) spectrum in the carbon $\mathrm{K}$ edge region for a film prepared at 2 mTorr, with a spectrum of glassy carbon shown for comparison. By calculating the area of the $1 \mathrm{~s}$ to $\pi^{*}$ feature at $284 \mathrm{eV}$, the fraction of $s p^{3}$ bonding in the HiPIMS film was determined to be $79 \%$, using the glassy carbon sample as a reference for $100 \% s p^{2}$ bonding. ${ }^{26}$ The inset in Figure 2(a) shows the spectrum from the 2 mTorr
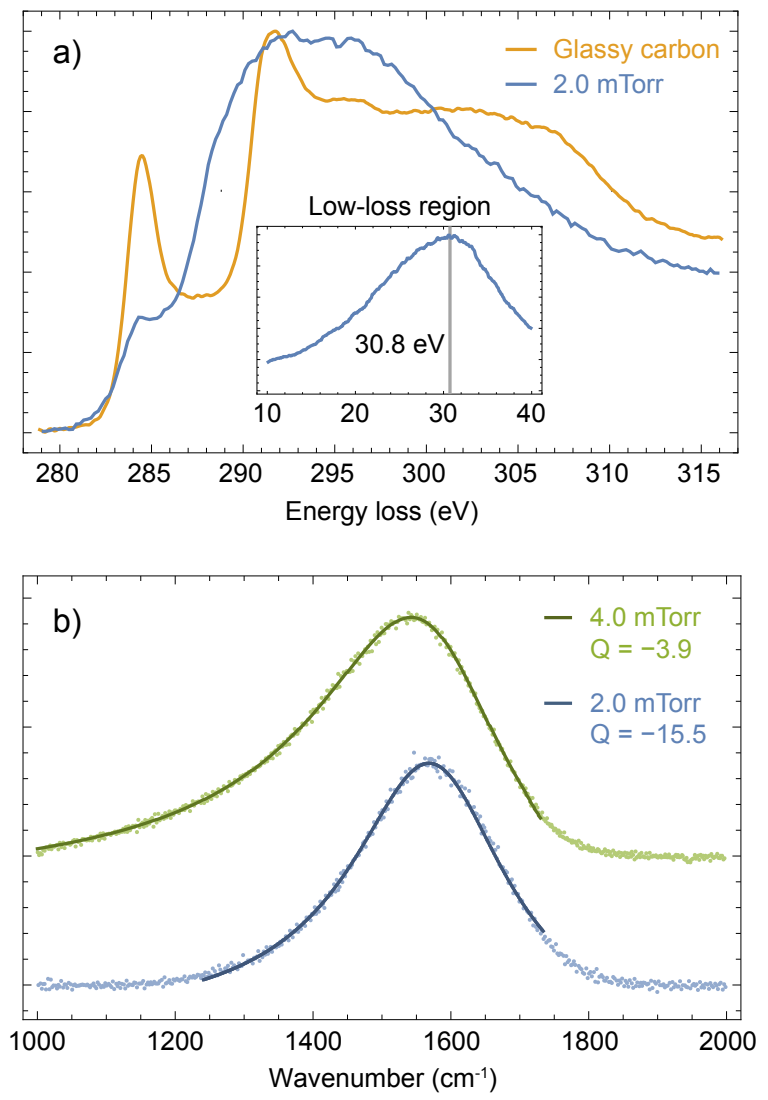

FIG. 2. (a) Electron energy loss spectra of glassy carbon and a mixed-mode HiPIMS film prepared at 2 mTorr. The inset shows the plasmon peak for the mixed-mode HiPIMS film. (b) Raman spectra of mixed-mode HiPIMS films deposited at pressures of 2 and 4 mTorr. The spectra have been fitted with a single Breit-Wigner-Fano line-shape (solid lines). sample in the low loss region, showing the plasmon peak is centered at $30.8 \mathrm{eV}$. Using a free-election model with an effective mass of $0.87 m_{e}$ for the plasmon frequency, ${ }^{27}$ this energy corresponds to a sample density of $3.0 \mathrm{~g} / \mathrm{cm}^{3}$.

Figure 2(b) shows the Raman spectra of films prepared at Ar pressures of 2 and 4 mTorr with fits to a Breit-Wigner-Fano (BWF) asymmetric Lorentzian line shape overlaid. Prior to fitting, the background of the spectrum and contribution from the Si peak signal were subtracted. Previous work ${ }^{28}$ has demonstrated that the degree of asymmetry is related to the $s p^{2}$ bonding fraction. The asymmetry can be quantified by the BWF fitting parameter $Q$, where a large negative value indicates a symmetrical line-shape and hence a higher $s p^{3}$ fraction. For the film prepared at 2 mTorr, a single highly symmetrical Raman peak $(\mathrm{Q}=-15.5)$ is observed, consistent with a large $s p^{3}$ fraction. The spectrum for the film prepared at 4 mTorr has an asymmetric line-shape $(\mathrm{Q}=-3.9)$, corresponding to a smaller $s p^{3}$ fraction.

The five film parameters plotted in Figure 3 demonstrate the effect of Ar pressure on the $s p^{3}$ fraction in the films. With increasing Ar pressure, a transition, highlighted in gray, occurs between tetrahedral and graphitic carbon. The longer mean free path at lower pressures means a significant fraction of carbon ions reach the substrate, as required for $s p^{3}$-bonded film growth. At higher pressures, the fraction of argon ions in the incident flux is higher, leading to peening of the surface and $s p^{2}$-dominated films. Previous work on mixed-mode deposition ${ }^{29}$ used a pressure of $5 \mathrm{mTorr}$, too high for the formation of $t a-\mathrm{C}$ according to our results, which may explain why ta-C was not observed in that work.

The first panel in Figure 3 shows the $s p^{3}$ fraction determined by fitting X-ray photoelectron spectroscopy (XPS) spectra (see Refs. 30 and 31 for methodology) with components corresponding to $s p^{2}$ bonding at $284.4 \mathrm{eV}, s p^{3}$ bonding at $285.2 \mathrm{eV}$ and much smaller contributions at higher energies corresponding to various oxidized carbon species. For the film prepared at 2 mTorr, the $s p^{3}$ fraction of $78 \%$ as measured by XPS is virtually identical to the value determined by EELS. The second panel shows the BWF line-shape parameter $\mathrm{Q}$ determined from the Raman spectra. The third panel shows the Tauc gap determined from ellipsometry measurements of the optical constants. At low pressures the Tauc gap is $2.7 \mathrm{eV}$, comparable to other amorphous carbon films with a high $s p^{3}$ content. ${ }^{32,33}$ The fourth panel shows the intrinsic compressive stress determined from wafer curvature and Stoney's equation. ${ }^{34}$ A compressive stress greater than $7 \mathrm{GPa}$ is associated with high $s p^{3}$ fraction, as seen in our previous cathodic arc studies ${ }^{35}$ where a transition to a diamond-like microstructure was observed at $6 \mathrm{GPa}$. The final panel shows the Ar content in the films as determined by XPS after using xenon to sputter away the surface layer. The maximum Ar content in the bulk region of the films is $1.25 \%$, lower than often observed for magnetron-sputtered films, and the only elements observed were $\mathrm{Ar}$ and $\mathrm{C}$. The minimum Ar content of $0.2 \%$ 


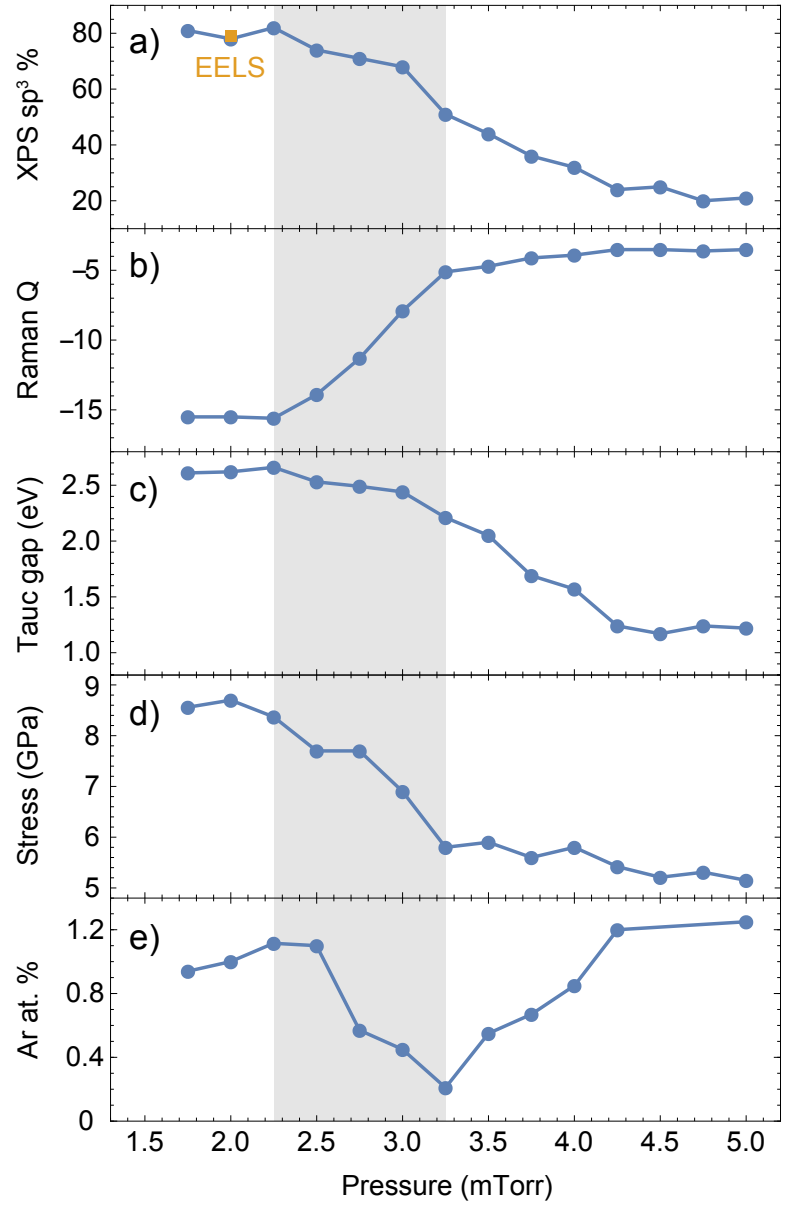

FIG. 3. Properties of carbon films deposited by mixed-mode HiPIMS as a function of Ar pressure. (a) $s p^{3}$ fraction determined by XPS; the gold square denotes the value obtained using EELS; (b) Raman Q factor obtained by fitting the spectra with a BWF line-shape as in Figure 2; (c) Tauc gap determined using ellipsometry; (d) compressive stress as measured by surface profilometry; and (e) Ar content determined by XPS after etching the surface with Xe.

occurs at intermediate Ar pressures, and is not monotically related to the process gas pressure. This behaviour is presumably linked to the large change in microstructure across the transition region. At pressures outside the transition region, argon incorporation decreases with decreasing Ar pressure as a result of a reduced flux of Ar ions relative to carbon ions.

In summary, we have demonstrated the formation of carbon films with a majority of $s p^{3}$ bonding using mixedmode HiPIMS implemented with a conventional magnetron sputtering system. Each pulse commences with a period of conventional HiPIMS operation after which a short-lived arc ignites on the cathode surface. This process increases the fraction of ionized carbon species and enables the formation of the tetrahedral phase via the application of substrate biasing. The maximum $s p^{3}$ fraction of $80 \%$ is very similar to values of $\sim 85 \%$ achieved with ion-beam techniques, and is robustly demonstrated by multiple lines of characterization (Raman lineshape, EELS, XPS, ellipsometry and stress measurements). Argon pressures below 2.5 mTorr were critical for obtaining films with a high $s p^{3}$ fraction. Above this pressure a transition occured to increasingly graphitic films. Since magnetron sputtering is a preferred method for commercial thin film deposition, this work opens up new applications for highly tetrahedral amorphous carbon films including VLSI-compatible devices, biosensors and optical devices.

\section{ACKNOWLEDGMENTS}

The authors gratefully acknowledge support from the Australian Research Council. N.A.M. acknowledges a fellowship under FT120100924. We thank M. Stueber and S. Ulrich of Karlsruhe Insitute of Technology for supplying the graphite targets. We also thank Dr Desmond Lau and Dr Matthew Field for their assistance in performing the EELS measurements and the facilities provided by the RMIT Microscopy \& Microanalysis Facility.

${ }^{1}$ S. Xu, B. K. Tay, H. S. Tan, L. Zhong, Y. Q. Tu, S. R. P. Silva, and W. I. Milne, J. Appl. Phys. 79, 7234 (1996).

${ }^{2}$ D. R. McKenzie, Rep. Prog. Phys. 59, 1611 (1996).

${ }^{3}$ S. Xu, D. Flynn, B. K. Tay, S. Prawer, K. W. Nugent, S. R. P. Silva, Y. Lifshitz, and W. I. Milne, Philos. Mag. B 76, 351 (1997).

${ }^{4}$ M. M. Golzan, D. R. McKenzie, D. J. Miller, S. J. Collocott, and G. A. J. Amaratunga, Diam. Relat. Mater. 4, 912 (1995).

${ }^{5}$ C. A. Davis, D. R. McKenzie, Y. Yin, E. Kravtchinskaia, G. A. J. Amaratunga, and V. S. Veerasamy, Philos. Mag. B 69, 1133 (1994).

${ }^{6}$ J. J. Cuomo, J. P. Doyle, J. Bruley, and J. C. Liu, Appl. Phys. Lett. 58, 466 (1991).

${ }^{7}$ J. Schwan, S. Ulrich, H. Roth, H. Ehrhardt, S. R. P. Silva, J. Robertson, R. Samlenski, and R. Brenn, J. Appl. Phys. 79, 1416 (1996).

${ }^{8}$ F. Bernhardt, K. Georgiadis, L. Dolle, O. Dambon, and F. Klocke, Mat.-wiss u. Werkstofftech. 44, 661 (2013).

${ }^{9}$ N.-H. Cho, K. M. Krishnan, D. K. Veirs, M. D. Rubin, C. B. Hopper, B. Bhushan, and D. B. Bogy, J. Mater. Res. 5, 2543 (1990).

${ }^{10}$ S. Logothetidis, M. Gioti, P. Patsalas, and C. Charitidis, Carbon 37, 765 (1999).

${ }^{11}$ K. Sarakinos, A. Braun, C. Zilkens, S. Mráz, J. M. Schneider, H. Zoubos, and P. Patsalas, Surf. Coat. Tech. 206, 2706 (2012).

${ }^{12}$ S. P. Bugaev, V. G. Podkovyrov, K. V. Oskomov, S. V. Smaykina, and N. S. Sochugov, Thin Solid Films 389, 16 (2001).

${ }^{13}$ B. M. DeKoven, P. R. Ward, R. E. Weiss, R. A. Christie, W. Scholl, D. Sproul, F. Tomasel, and A. Anders, Society of Vaccum Coaters. 46th Annual Technical Conference Proceedings , 158 (2003).

${ }^{14}$ L. A. Donohue, A. Torosyan, P. May, D. E. Wolfe, K. J, and E. T. J, Plat. Surf. Finish. , 38 (2009).

${ }^{15}$ S. Nakao, K. Yukimura, H. Ogiso, S. Nakano, and T. Sonoda, Vaccum 89, 261 (2013).

${ }^{16}$ M. Hiratsuka, A. Azuma, H. Nakamori, Y. Kogo, and K. Yukimura, Surf. Coat. Tech. 229, 46 (2013).

${ }^{17}$ K. Yukimura, H. Ogiso, S. Nakano, S. Nakao, and K. Takaki, IEEE T. Plasma Sci. 41, 3012 (2013).

${ }^{18}$ M. Huang, X. Zhang, P. Ke, and A. Wang, Appl. Surf. Sci. 283, 321 (2013).

${ }^{19}$ J. Lin, W. D. Sproul, R. Wei, and R. Chistyakov, Surf. Coat. Tech. 258, 1212 (2014). 
${ }^{20}$ A. Aijaz, K. Sarakinos, D. Lundin, N. Brenning, and U. Helmersson, Diam. Relat. Mater. 23, 1 (2012).

${ }^{21}$ M. Lattemann, A. Moafi, M. M. M. Bilek, D. G. McCulloch, and D. R. McKenzie, Carbon 48, 918 (2010).

${ }^{22}$ A. Anders, Cathodic Arcs: From Fractal Spots to Energetic Condensation, 1st ed., Springer Series on Atomic, Optical, and Plasma Physics, Vol. 50 (Springer-Verlag New York, 2008).

${ }^{23}$ B. Jüttner and I. Kleberg, J. Phys. D: Appl. Phys. 33, 2025 (2000).

${ }^{24}$ C. W. Kimblin, J. Appl. Phys. 44, 3074 (1973).

${ }^{25}$ A. P. Ehiasarian, A. Hecimovic, T. de los Arcos, R. New, V. Schulz-von der Gathen, M. Boke, and J. Winter, Appl. Phys. Lett. 100, 114101 (2012).

${ }^{26}$ Bosman, $\mathrm{M}$ and Keast, $\mathrm{V}$ J and Watanabe, $\mathrm{M}$ and McCulloch, D G and Shakerzadeh, M and Teo, E H T and Tay, B K, Carbon 47, 94 (2009).

${ }^{27}$ A. C. Ferrari, A. Libassi, B. K. Tanner, V. Stolojan, J. Yuan, L. M. Brown, S. E. Rodil, B. Kleinsorge, and J. Robertson,
Phys. Rev. B 62, 11089 (2000).

${ }^{28}$ S. Prawer, K. W. Nugent, Y. Lifshitz, G. D. Lempert, E. Grossman, J. Kulik, I. Avigal, and R. Kalish, Diam. Relat. Mater. 5, 433 (1996).

${ }^{29}$ M. Lattemann, B. Abendroth, A. Moafi, D. G. McCulloch, and D. R. McKenzie, Diam. Relat. Mater. 20, 68 (2011).

${ }^{30}$ J. Díaz, G. Paolicelli, S. Ferrer, and F. Comin, Phys. Rev. B 54, 8064 (1996).

${ }^{31}$ P. Mérel, M. Tabbal, M. Chaker, S. Moisa, and J. Margot, Appl. Surf. Sci. 136, 105 (1998).

${ }^{32}$ J. Lee, R. W. Collins, V. S. Veerasamy, and J. Robertson, Diamond And Related Materials 7, 999 (1998).

${ }^{33}$ K. B. K. Teo, A. C. Ferrari, G. Fanchini, S. E. Rodil, J. Yuan, J. T. H. Tsai, E. Laurenti, A. Tagliaferro, J. Robertson, and W. I. Milne, Diam. Relat. Mater. 11, 1086 (2002).

${ }^{34}$ G. G. Stoney, Proc. Roy. Soc. A 82, 172 (1909).

${ }^{35}$ D. W. M. Lau, D. G. McCulloch, M. B. Taylor, J. G. Partridge, D. R. McKenzie, N. A. Marks, E. H. T. Teo, and B. K. Tay, Phys. Rev. Lett. 100, 4 (2008). 